\title{
Influência da Terapia por Contensão Induzida em Indivíduos hemiparéticos após acidente vascular encefálico: revisão sistemática de literatura
}

O Acidente Vascular Encefálico é uma das maiores causas de limitação funcional e morte no mundo. Diversos métodos de intervenção são utilizados para a reabilitação de hemiparéticos em decorrência de Acidente Vascular Encefálico e um dos mais recentes é a Terapia por Contensão Induzida. Revisar sistematicamente as evidências sobre a influência da Terapia por Contensão Induzida como intervenção em indivíduos hemiparéticos com Acidente Vascular Encefálico. Foram realizadas pesquisas de abril a junho de 2011 nas bases de dados Pubmed, Scielo e PEDro, utilizando-se os unitermos "hemiplegic", "stroke" e "physical therapy". Os estudos selecionados foram publicados na língua inglesa, no período de 2000 a 2010, utilizaram a Terapia por Contensão Induzida em indivíduos com Acidente Vascular Encefálico e obtiveram pontuação mínima de cinco pontos na escala PEDro. Dos 44 estudos encontrados, 27 foram incluídos na revisão. Destes manuscritos, o número médio de participantes variou de 30 a 45 indivíduos e a faixa etária foi de 23 a 88 anos. Os instrumentos de avaliação mais utilizados foram o Motor Activity Log e o Wolf Motor Function Test. A Terapia por Contensão Induzida mostrou-se eficaz em indivíduos com Acidente Vascular Encefálico que apresentam déficits funcionais de membro superior.

Palavras-chave: Hemiplegia; Acidente Vascular Encefálico; Reabilitação.

\section{Influence of the Constraint-Induced Movement Therapy in hemiparetic patients stroke: a systematic literature review}

Stroke is one the most cause of functional limitation and mortality. Several methods of intervention are used for rehabilitation of hemiparetic as a result of stroke, and one the most recent is the Constraint-Induced Movement Therapy. To review systematically the evidence on the influence of Constraint-Induced Movement Therapy as intervention in hemiparetic individuals stroke. A search was conducted during the period April to June 2011 in databases PubMed, Scielo and PEDro, using the keywords "hemiplegic", "stroke" and "physical therapy". The selected studies were published in English in the period 2000 to 2010 , used ConstraintInduced Movement Therapy in individuals stroke and have obtained minimum score of five points in Pedro's scale. Of the 44 studies found, 27 were included in the review. Of these manuscripts, the average number of participants ranged from 30 to 45 individuals and the age range was 23 to 88 years. The assessment tools most frequently used were the Motor Activity Log and Wolf Motor Function Test. Constraint-Induced Movement Therapy was effective in individuals stroke who have functional deficits of the upper limb.

Keywords: Hemiplegic; Stroke; Rehabilitation.

Topic: Fisioterapia

Reviewed anonymously in the process of blind peer.

Diogo Costa Garção

Universidade Federal de São Carlos, Brasil

diogoufscar@yahoo.com.br

Akeline Santos de Almeida

Universidade Federal de Sergipe, Brasil

akeline se@hotmail.com

Aline Jorge Rodrigues Sobral

Universidade Federal de Sergipe, Brasil

linesobral@hotmail.com

Dayane Evellyn dos Santos

Universidade Federal de Sergipe, Brasil

dayane_evellyn@hotmail.com

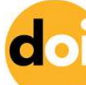

DOI: 10.6008/SPC2236-9600.2016.001.0003
Received: 05/05/2016

Approved: 11/08/2016

\author{
Juliana de Santana Santos \\ Universidade Federal de Sergipe, Brasil
}

juli-santos2008@bol.com.br

Anne Caroline Santos

Universidade Tiradentes, Brasil

carolineefisio@hotmail.com

Tássia Virginia de Carvalho Oliveira

Universidade Tiradentes, Brasil

http://lattes.cnpq.br/2273361044731342

tassinhafisio@yahoo.com.br

Referencing this:

GARÇÃO, D. C.; ALMEIDA, A. S.; SOBRAL, A. J. R.; SANTOS, D. E.; SANTOS, J. S.; SANTOS, A. C.; OLIVEIRA, T. V. C.. Influência da Terapia por Contensão Induzida em Indivíduos hemiparéticos após acidente vascular encefálico: revisão sistemática de literatura. Scire Salutis, v.6, n.1, p.52-63, 2016. DOI: http://doi.org/10.6008/SPC22369600.2016.001.0003 


\section{INTRODUÇÃO}

O Acidente Vascular Encefálico (AVE) é uma das maiores causas de limitação funcional e morte no mundo, com destaque para os países subdesenvolvidos e emergentes. No Brasil, o AVE é a principal causa de morte e incapacidade funcional e as taxas de mortalidade ajustadas à idade estão entre as maiores em nove países da América Latina constituindo, desse modo, um importante problema de saúde pública (TORRIANI et al., 2005; MAIKI et al., 2005).

O AVE é definido como uma lesão encefálica decorrente do comprometimento no fluxo sanguíneo local por danos às artérias que irrigam o encéfalo. Tais danos podem ser causados tanto por obstrução de uma artéria, caracterizando o AVE isquêmico, como por ruptura de um vaso, caracterizando o AVE hemorrágico (TORRIANI et al., 2005; SCHUSTER et al., 2008; ESCARCEL et al., 2010).

O tipo isquêmico pode ocorrer como resultado de trombos ou problemas que causem baixas pressões de perfusão sistêmica. Enquanto o tipo hemorrágico acontece quando há um sangramento anormal, causado por aneurisma ou trauma, para dentro das áreas extravasculares do cérebro. A hemorragia resulta em um aumento das pressões intracranianas, lesionando os tecidos cerebrais e restringindo o fluxo sanguíneo distal (LEITE et al., 2009).

Tais distúrbios geram comprometimentos motores que podem vir associados a alterações sensitiva, mental, perceptiva, visual e da linguagem. A severidade do quadro clínico dependerá diretamente da localização da lesão, do tamanho da área encefálica atingida e do tempo que o paciente esperou para ser socorrido (TORRIANI et al., 2005; TORRIANI et al., 2008; MAKI et al., 2005; MACEDO, 2008; TEIXEIRA-SALMELA et al., 2000).

Os comprometimentos motores são os mais evidentes e, geralmente, constituem-se a principal queixa de indivíduos após um quadro de AVE. Estes cursam com hemiplegia ou hemiparesia que se manifestam como perda ou dificuldade, respectivamente, de mobilidade no tronco e nas extremidades levando a estratégias compensatórias do hemicorpo afetado e à perda da independência na vida diária.

O maior impacto ocorre na função do membro superior uma vez que a lesão resulta em controle ineficaz dos movimentos do braço e redução da destreza manual e digital (TEIXEIRA-SALMELA et al., 2005; SCHUSTER et al., 2008; SANTOS et al., 2008). Diversos métodos de intervenção são utilizados para a reabilitação de indivíduos hemiparéticos decorrentes de AVE, como conceito Bobath, método FNP Facilitação Neuromuscular Proprioceptiva -, método Rood e método Brunnstrom (CARVALHO, 2007).

Recentemente uma técnica proposta para a reabilitação de pacientes pós-AVE é a Terapia por Contensão Induzida (TCl), a qual enfatiza a recuperação das habilidades funcionais do membro superior, comprometido por lesões encefálicas que afetam apenas um hemídio corporal, e facilita o retorno gradual às atividades de vida diária de forma mais independente, uma vez que intensifica o treino funcional (SILVA, 2010; RIBERTO et al., 2005).

A TCl caracteriza-se pela contensão do membro menos comprometido, por meio de luvas, tipoias ou slings, associada a treino intensivo de utilização do membro oposto. $O$ treino funcional é baseado na seleção de 
tarefas que simulam as atividades de vida diária, as quais são adequadas às capacidades individuais e sofrem graduação de dificuldade e complexidade. Desse modo a TCl permite um treino mais funcional e dinâmico quando comparada às técnicas tradicionais, o que otimiza os ganhos e a evolução do paciente (BRANDÃO et al., 2009)

A intervenção foi desenvolvida por Edward Taub em 1980 a partir da observação de que primatas submetidos à lesão em um dos membros superiores não utilizavam esta extremidade em suas atividades diárias, fenômeno conhecido como "desuso aprendido". No entanto, após ser aplicada uma restrição no membro menos comprometido, os animais foram forçados a utilizar o membro mais comprometido nas atividades cotidianas, o que provocou a superação do desuso aprendido, comprovada por exames de neuroimagem que evidenciaram uma reorganização cortical, ou seja, a formação de sinapses adicionais (BRANDÃO, 2007).

O protocolo original da TCl inclui a contensão do membro menos comprometido durante $90 \%$ do dia e o treino intensivo do membro oposto por um período de seis horas diárias durante duas ou três semanas. Porém, em muitos estudos esse protocolo foi modificado haja vista os pacientes relatarem incômodo e cansaço excessivo devido aos tempos prolongados de contensão e treino funcional. Assim, embora os resultados mostrem-se promissores, a TCl pode ser de difícil implantação em algumas situações clínicas (BRANDÃO, 2007).

Esse estudo teve como objetivo revisar sistematicamente diferentes abordagens da TCl e padronizar um possível protocolo de intervenção.

\section{METODOLOGIA}

Foram realizadas pesquisas durante o período de abril a junho de 2011 nas bases de dados eletrônicas Pubmed, Scielo e PEDro, utilizando-se os unitermos "hemiplegic", "stroke" e "physical therapy". A busca de artigos foi realizada em três idiomas, inglês, português e espanhol, independente de idade, gênero ou qualquer outro grupo.

Os artigos resultantes do levantamento bibliográfico foram analisados por quatro pesquisadores com base nos seguintes critérios de inclusão: (1) trabalhos publicados em inglês no período de 2000 a 2010, (2) estudos realizados em pacientes com AVE e (3) pontuação mínima de cinco pontos na escala PEDro (PEDro 2011).

A escala PEDro foi desenvolvida pela Physiotherapy Evidence Database para ser empregada em estudos experimentais e consiste em onze itens com duas opções de respostas, "sim" ou "não", e a somatória de respostas "sim" é a pontuação final. Como o item 1 não é pontuado, a escala tem uma pontuação total de até 10 pontos. Os itens são: 1) especificação dos critérios de inclusão (item não pontuado); 2) alocação aleatória; 3) sigilo na alocação; 4) similaridade dos grupos na fase inicial ou basal; 5) mascaramento dos sujeitos; 6) mascaramento do terapeuta; 7) mascaramento do avaliador; 8) medida de pelo menos um desfecho primário em $85 \%$ dos sujeitos alocados; 9) análise da intenção de tratar; 10) comparação entre grupos de pelo menos um desfecho primário e 11) relato de medidas de variabilidade e estimativa dos 
parâmetros de pelo menos uma variável primária (PEDro 2011). Os detalhes de cada item podem ser observados na tabela I.

Os pesquisadores avaliaram independentemente cada artigo com relação à presença ou ausência dos indicadores de qualidade da escala. Níveis moderados de confiabilidade entre examinadores (ICC $=0,68$; IC95\%=0,57-0,76) têm sido demonstrados pela escala PEDro. As discordâncias entre os pesquisadores foram resolvidas por discussão e consenso.

Após o levantamento bibliográfico e a análise dos estudos encontrados, os artigos selecionados para a revisão foram resumidos de forma padronizada com base nos seguintes tópicos: objetivos do estudo, critérios de inclusão e exclusão, características dos participantes, instrumentos de avaliação utilizados e protocolo de intervenção. Em seguida, os pesquisadores correlacionaram o conteúdo dos trabalhos a fim de identificar as práticas de intervenção e suas variações.

\section{RESULTADOS}

Resultaram da busca inicial por meio dos unitermos 44 artigos com características passíveis de entrarem na revisão. No entanto, 17 estudos foram excluídos por não cumprirem os critérios de inclusão, uma vez que a publicação não compreendeu o período de 2000 a 2010 (n=2), a amostra não era composta por pacientes pós-AVE $(n=1)$ e a pontuação na escala PEDro foi inferior a 5 pontos $(n=7)$. Além disso, foram excluídas as pesquisas caracterizadas como Estudos de Caso $(n=4)$, Série de Casos $(n=3)$ ou Revisão Sistemática de Literatura $(n=1)$. Assim, 27 trabalhos apresentaram os critérios determinados pelos pesquisadores e foram selecionados para a análise do conteúdo. O resumo das principais características destes estudos é apresentado na tabela II.

\section{Objetivos do Estudo}

A maioria dos estudos analisados avaliaram a eficácia da $\mathrm{TCI}$ na recuperação da funcionalidade do membro superior mais comprometido por meio de comparação com a terapia tradicional (WOLF et, 2006; DAHL et al., 2008) ou com o treinamento bilateral (LIN et al., 2009). No entanto, seis estudos (WU, 2007 et al.; Underwood, 2006) realizaram somente a comparação entre os períodos pré e pós-intervenção de indivíduos submetidos à TCl, sem a utilização de Grupo Controle ou outro protocolo.

Além de avaliar se a TCI tem a capacidade de anular os efeitos causados pelo AVE no desempenho funcional do membro superior, Gauthier et al. (2009) analisaram se o local da lesão encefálica influenciava os ganhos motores pós-intervenção (UNDERWOOD et al., 2009). Por outro lado, Broghard et al. (2009) investigaram a influência do uso de uma luva para contensão da extremidade menos comprometida sobre os resultados da TCI (BROGÅRDH et al., 2009).

Haja vista grande parte dos estudos sobre a TCl serem realizados em pacientes com AVE crônico, alguns trabalhos tiveram como objetivo investigar a influência da intervenção nas fases aguda (Boake, 2007) e subaguda da lesão (WINSTEIN et al., 2003; MYIN, 2008). Wolf et al. (2010) avaliaram a eficácia da TCI 
aplicada no período precoce do AVE (3-9meses) em comparação com o período tardio (15-21 meses) (WOLF, 2010).

Adaptações nas técnicas da $\mathrm{TCl}$ foram realizadas em seis dos artigos analisados, os quais tiveram como objetivos comparar a TCl tradicional versus a TCI modificada (WITTENBERG et al. , 2003) investigar a eficácia de um protocolo modificado de TCl (sem restrição física) (YEN et al., 2005), avaliar a efetividade de um dispositivo que automatiza a TCI (TAUB et al., 2005), investigar os benefícios da TCl modificada (tempo curto) na fase subaguda pós-AVE (BROGÅRDH et al., 2010), investigar os efeitos da TCl quando associado a um medicamento (Donepezil) na melhora da funcionalidade da extremidade superior (NADEAU et al., 2004) e avaliar a TCl associada à prática mental (PM) em todas as fases pós-AVE (PAGE, 2009).

A PM consiste em ensaio cognitivo, realizado na ausência de movimentos voluntários, fornecido imediatamente após as sessões de TCl. A PM dura trinta minutos em ambiente tranquilo e suas atividades correspondem às praticadas durante a intervenção clínica. São executados, de início, cerca de 5 minutos de exercícios relaxantes guiados, em seguida, 15-20 minutos de imagética motora guiada, na qual o sujeito é instruído a imaginar a si mesmo na execução de cada atividade funcional realizada durante a TCl e, finalmente, 5 minutos de reorientação em sala (PAGE, 2009).

\section{Critérios de Inclusão e Exclusão}

Nos artigos estudados foram estabelecidos critérios de inclusão e exclusão com o objetivo de tornar a amostra de sujeitos o mais semelhante possível e assim garantir a homogeneidade. Dessa forma, participaram da intervenção indivíduos que obedeceram aos seguintes critérios de inclusão: possuir capacidade cognitiva preservada para compreender comandos verbais simples e executar as atividades realizadas na intervenção (WOLF et, 2006; TAUB, USWATTE et al., 2006; BROGÅRDH et al., 2009; WOLF , 2010; YEN et al., 2005; BROGÅRDH et al., 2010; PAGE et al., 2009) , apresentar amplitude para extensão ativa de no mínimo $10^{\circ}$ nas articulações metacarpofalangiana e interfalangianas e $20^{\circ}$ na articulação do punho (WOLF et, 2006; WU et al. 2007; DAHL et al., 2008; PAGE et al., 2004; UNDERWOOD et al., 2006; BROGÅRDH et al., 2009; WINSTEIN et al., 2003; PAGE et al., 2009), hemiparesia persistente (BOAKE et al., 2007; MYINT et al., 2008; MYINT et al., 2008) estabilidade suficiente para andar quando o membro superior menos comprometido estiver contido (WOLF et al., 2006; WU et al., 2007; LIN et al., 2009; WU, CHEN et al., 2007; SUPUTTITADA et al., 2004; TAUB, 2005; USWATTE et al., 2006; BROGÅRDH et al., 2009; MYINT et al., 2008; MYINT et al., 2008; TAUB et al., 2005; BROGÅRDH et al., 2010), desuso aprendido considerável (pontuação <2,5 na MAL) (WOLF et al., 2006; WU et al., 2007; LIN et al., 2009; WU, CHEN et al., 2007; PAGE et al., 2008; TAUB, USWATTE et al., 2006; WITTENBERG et al., 2003; TAUB et al., 2005; NADEAU et al., 2004; PAGE et al., 2009) e ter sofrido somente um AVE (SUPUTTITADA et al., 2004; PAGE et al., 2008; BROGÅRDH et al., 2009; WINSTEIN et al., 2003; YEN et al., 2005; BROGÅRDH et al., 2010; PAGE et al., 2009).

Quanto aos critérios de exclusão, foram utilizados aqueles que dificultavam a realização da terapia pelo sujeito, como dor intensa no membro superior, medida pelo escore de 4 pontos ou mais em um total de 10 pontos da escala visual analógica da dor (EVA) (WOLF et al., 2006; PAGE et al., 2008; TAUB et al., 2006 
USWATTE et al., 2006; MYINT et al., 2008; MYINT et al., 2008; TAUB et al., 2005; PAGE et al., 2009), presença de espasticidade severa (WOLF et al., 2006; WU et al. 2007; LIN et al., 2009; PAGE et al., 2008; TAUB et al., 2005; USWATTE et al., 2006; TAUB et al., 2005; PAGE et al., 2009), participação em outro estudo de intervenção motora com uso de toxina botulínica e em período menor que um ano (WOLF et al., 2006; WU et al., 2007; PAGE et al., 2008; BROGÅRDH et al., 2009; WINSTEIN et al., 2003; WOLF, 2010; PAGE et al., 2009), deformidades no membro superior mais comprometido decorrentes de fraturas ou patologias diversas (DROMERICK et al., 2000; DROMERICK et al., 2009; BROGÅRDH et al., 2009; WINSTEIN et al., 2003; BROGÅRDH et al., 2010) e afasia grave (SUPUTTITADA et al., 2004; TAUB 2005; USWATTE et al., 2006; MYINT et al., 2008; YEN et al., 2005; BROGÅRDH et al., 2010).

\section{Características dos Participantes}

Os pacientes foram recrutados e incluídos no programa de intervenção mediante o preenchimento dos critérios de inclusão pré-estabelecidos. O número médio de participantes variou de 30 (WU et al., 2007; DAHL et al., 2008; YEN et al., 2005). 42 a 45 indivíduos (WU et al., 2007) e a faixa etária predominante foi de 23 (WU, CHEN et al., 2007) a 88 anos (GAUTHIER et al., 2009). As amostras eram constituídas de sujeitos de ambos os gêneros e verificou-se que a terapia teve início entre duas semanas (DAHL et al., 2008; BOAKE et al., 2007; MYINT et al., 2008; MYINT et al., 2008) e oito anos após a identificação dos déficits motores (DAHL et al., 2008), com maior prevalência da faixa de tempo de três meses (WU et al., 2007; UNDERWOOD et al., 2006; BROGÅRDH et al., 2009; WINSTEIN et al., 2003; WOLF, 2010) a um ano (WOLF et al., 2006; WU et al., 2007; PAGE et al., 2004; UNDERWOOD et al., 2006; TAUB et al., 2005; NADEAU et al., 2004).

\section{Instrumentos de Avaliação Padronizados}

Todos os estudos utilizaram Instrumentos de Avaliação Padronizados a fim de avaliar a função motora do membro superior dos participantes antes e após a intervenção e, desse modo, quantificar e/ou qualificar os resultados obtidos. Entre os mais utilizados estão:

Motor Activity Log (MAL) - compreende uma entrevista de 14 itens para hemiparéticos que sofreram AVE e tem como objetivo avaliar o uso espontâneo do braço e mão paréticos durante as atividades de vida diária do indivíduo, utilizando-se uma escala que varia de 0 a 5 pontos. Assim, considera o desuso aprendido e a requisição funcional do membro superior mais comprometido nas atividades de vida diária (SALIBA et al., 2008). Foi utilizado em 21 dos estudos analisados (WOLF et al., 2006; WU et al., 2007; TAUB et al., 2005; USWATTE et al., 2006; GAUTHIER et al., 2009; WITTENBERG et al., 2003; TAUB et al., 2005; NADEAU et al., 2004).

Wolf Motor Function Test (WMFT) - foi desenvolvido a partir da união e modificação dos testes FuglMeyer, TEMPA, Escala de Habilidade Funcional (FAS) e teste de Habilidade Motora para Mão (AMAT). Consiste em uma ferramenta de fácil aplicação, com 15 tarefas funcionais e uma escala de graduação de 0 a 5 pontos, que avalia a função motora da extremidade superior. O teste, específico para hemiplégicos, é validado e bastante fidedigno (MARCOTTI et al., 2005). Observou-se sua utilização em 12 do total de estudos 
analisados (WOLF et al., 2006; RICHARDS et al., 2006; DAHL et al., 2008; TAUB et al., 2005, USWATTE et al., 2006; GAUTHIER et al., 2009; WINSTEIN et al., 2003; WOLF, 2010; NADEAU et al., 2004).

Fugl Meyer Assessment (FMA) - é um sistema de pontuação numérica acumulativa que avalia seis aspectos do paciente: amplitude de movimento, dor, sensibilidade, função motora da extremidade superior e inferior, equilíbrio, além da coordenação e velocidade (MENEGHETTI et al., 2010). Foi aplicada em 9 do total de estudos analisados (WU et al., 2007; LIN et al., 2009; PAGE et al., 2004; PAGE et al., 2008; UNDERWOOD et al., 2006; BOAKE et al., 2007; PAGE et al., 2009).

Action Research Arm Test (ARAT) - composto por 19 itens, é uma versão resumida da escala "Função da extremidade superior" que avalia as atividades funcionais da extremidade superior sobre o pressuposto de que estas podem ser sintetizadas em quatro tipos básicos de função: compressão, preensão, pinçamento e atividades de alcance (função motora grossa). Nos sub-testes compressão e pinçamento, o paciente sentado em frente a uma mesa deve apreender objetos (por exemplo, cubos de tamanhos diferentes) e distribuí-los em uma prateleira colocada sobre a mesma. Além disso, são requisitadas atividades de alcance, como colocar a mão atrás da cabeça, e outras tarefas, como passar água de um copo para outro (Paz et al., 2007; Paz et al., 2007). Observou-se sua aplicação em 8 dos estudos analisados (DROMERICK et al., 2000; DROMERICK et al., 2009; PAGE et al., 2004; PAGE et al., 2008; MYINT et al., 2008; PAGE et al., 2009).

Um dos estudos, além de aplicar o ARAT na avaliação funcional da extremidade superior, utilizou o dinamômetro com a finalidade de quantificar a força de preensão manual (SUPUTTITADA et al., 2008). Esse instrumento possui uma alça fixa e outra móvel, um painel com unidades em libras/quilograma força e um sistema hidráulico fechado capaz de mensurar a força produzida por uma contração isométrica aplicada sobre as alças. De acordo com as recomendações da American Society of Hand Therapy, o indivíduo deve ser posicionado na postura sentada em uma cadeira com encosto, sem apoio para os braços, ombro aduzido e neutramente rodado, cotovelo flexionado a 90ㅡ, antebraço em posição neutra, punho entre 0 e 30 de extensão e 0 ㅇ a 15으 de desvio ulnar. Os escores são obtidos através da média de três tentativas, com intervalo de repouso de 60 segundos entre as mesmas (Fess, 1992).

\section{Protocolo de Intervenção}

O protocolo original da TCI foi utilizado em 8 dos estudos analisados (WOLF et al., 2006; DAHL et al., 2008; SUPUTTITADA et al., 2004; TAUB et al., 2005; USWATTE et al., 2006; GAUTHIER et al., 2009; WINSTEIN CJ et al., 2003; WOLF et al., 2010). No entanto, 17 estudos modificaram tal protocolo (DROMERICK et al., 2000; WU et al., 2007; LIN et al., 2009; PAGE et al., 2004; PAGE et al., 2008; BROGÅRDH et al., 2009; BOAKE et al., 2007; MYINT et al., 2008; MYINT et al., 2008; WITTENBERG et al., 2003; BROGÅRDH et al., 2010; PAGE et al., 2009). Principalmente quanto ao tempo da terapia, que variou de meia-hora a cinco horas diárias (DROMERICK et al., 2000; WU et al., 2007; LIN et al., 2009; PAGE et al., 2004; PAGE et al., 2008; BROGÅRDH et al., 2009; BOAKE et al., 2007; MYINT et al., 2008; WITTENBERG et al., 2003; TAUB et al., 2005; BROGÅRDH et al., 2010; PAGE et al., 2009) e/ou de uso da contensão, a qual foi retirada (YENG et al., 2005) ou teve sua utilização reduzida para três a seis horas diariamente (DROMERICK et al., 2000; WU et al., 2007; LIN et al., 
2009; PAGE et al., 2004; PAGE et al., 2008; WITTENBERG et al., 2003). Em dois desses trabalhos (TAUB et al., 2005; PAGE et al., 2009) a TCI foi associada a outras intervenções, como PM e automatização da terapia.

Nos demais artigos, os autores utilizaram medicamentos (Donepezil) em associação à $\mathrm{TCl}$ a fim de avaliar a melhora na funcionalidade do membro superior mais comprometido de indivíduos pós-AVE (RICHARDS et al., 2006; NADEAU et al., 2004). Richards et al. (2006) e Nadeau et al. (2004) administraram 1 comprimido por dia $(30 \mathrm{mg}$ ) durante duas semanas e, em seguida, 2 comprimidos por dia durante quatro semanas. Nas duas últimas semanas os indivíduos foram submetidos ao protocolo original da TCl.

Diversos estudos compararam a prática da $\mathrm{TCl}$ com um Grupo Controle, o qual, na maioria das vezes, foi submetido à terapia convencional. Esta tem por base técnicas de Facilitação Neuromuscular Proprioceptiva (FNP) e treino de tarefas funcionais e atividades compensatórias com o membro menos comprometido (WOLF et al., 2006; DAHL et al., 2008).

\section{DISCUSSÃO}

Tendo em vista todos os estudos analisados, constatou-se, com base em evidências científicas, que a $\mathrm{TCl}$ se mostrou eficaz no tratamento dos déficits no membro superior de pacientes com hemiparesia causada por Acidente Vascular Encefálico (AVE) (WOLF et al., 2006; PAGE et al., 2009). Os estudos relataram ganhos significativamente maiores nos indivíduos submetidos à $\mathrm{TCl}$ quando em comparação com sujeitos tratados por meio da terapia convencional através de técnicas do neurodesenvolvimento, alongamento do membro acometido e treino de habilidades motoras finas (WOLF et al., 2006; DAHL et al., 2008).

Taub et al. (2006) aplicaram a TCl em um grupo de 21 participantes a fim de verificar sua eficácia através da comparação com um grupo placebo controlado composto por 20 pacientes (TAUB et al., 2005; USWATTE et al., 2006). Em seus resultados encontraram melhorias significativas na habilidade e funcionalidade do membro superior mais comprometido no grupo $\mathrm{TCl}$ em relação ao grupo placebo (TAUB et al., 2005; USWATTE et al., 2006). Tal dado corrobora o estudo de Lin et al. (2009) que, com o objetivo de investigar os efeitos da $\mathrm{TCl}$ em comparação com o treinamento bilateral, demonstrou maiores ganhos na função diária, uso funcional do braço afetado e qualidade de vida no grupo de pacientes submetidos a TCI (LIN et al., 2009).

Brandão (2007) cita que os efeitos do protocolo de tarefas específicas da TCI na função do membro superior parecem estar ligados à reorganização cortical uso-dependente e à superação do fenômeno descrito como desuso aprendido (BRANDÃO, 2007). A reorganização cortical, observada por exames de neuroimagem realizados antes e após a intervenção, demonstra que a utilização de padrões de movimento repetitivo do braço afetado é capaz de provocar mudanças estruturais e funcionais no sistema nervoso central em virtude da formação de sinapses adicionais e do aumento da eficiência da transmissão sináptica (BRANDÃO, 2007).

A superação do desuso aprendido decorre essencialmente do treino intensivo do membro mais comprometido, que aumenta a motivação e contingência do uso dessa extremidade e favorece ganhos de habilidades motoras importantes para o retorno da função do membro (BRANDÃO, 2007). Em seu estudo, Page et al. (2004) aplicaram a TCl em um grupo de pacientes com AVE crônico e concluíram que a mesma é 
um método eficaz para melhorar a função do membro superior mais comprometido desses indivíduos. Para tanto, os autores enfatizam que o treino intensivo é de fundamental importância na reaquisição das habilidades funcionais (PAGE et al., 2004).

O protocolo original da TCl inclui a contensão do membro menos comprometido durante $90 \%$ do dia associada a treino intensivo do membro oposto por um período de 6 horas diárias durante duas ou três semanas (BRANDÃO, 2007). Wolf et al. (2006) utilizaram tal protocolo em seu estudo, sendo que a aderência à contensão extra laboratório foi monitorada por um sensor físico e um timer colocados na luva bem como um diário de atividades para casa (WOLF et al., 2006). A terapia mostrou-se eficaz haja vista os participantes obtiveram ganhos significativos de função e habilidades motoras do membro superior mais comprometido e as melhoras persistiram por até um ano após o final da intervenção. Segundo os pesquisadores, tais ganhos não foram influenciados pela idade, sexo ou nível inicial da função do braço (WOLF et al., 2006).

Diversos estudos mostraram que os benefícios da TCl independem do estágio de evolução da doença (UNDERWOOD et al., 2006; WINSTEIN et al., 200; WOLF, 2010). Utilizando-se do protocolo supracitado, tais estudos não encontraram diferenças estatisticamente significativas nos ganhos motores após TCl quando comparados indivíduos nas fases subaguda e crônica da lesão. Underwood et al. (2006) usaram tal protocolo em 18 indivíduos na fase subaguda (3-9 meses da lesão) e 14 sujeitos na fase crônica (mais de 1 ano da lesão) (UNDERWOOD et al., 2006). Em seus resultados não encontraram diferenças na dor, fadiga e função manual, ou seja, os sujeitos de ambos os grupos foram capazes de tolerar o mesmo nível de terapia (UNDERWOOD et al., 2006).

Dromerick et al. (2000) realizou um estudo piloto a fim de testar a eficácia da TCl durante o processo de reabilitação aguda de pacientes pós AVE por meio da comparação com um grupo controle, que recebeu apenas a terapia tradicional para ganho de força e amplitude de movimento dos membros superiores (DROMERICK et al., 2000). O protocolo de TCl utilizado foi de contensão diária de 6 horas, apenas durante a intervenção, por um período de 14 dias. A TCl mostrou-se eficaz durante a terapia de reabilitação aguda tendo em vista que os pacientes do grupo experimental tiveram melhoras significativas de função e habilidades motoras do membro superior mais comprometido quando em comparação com o grupo controle (DROMERICK et al., 2000).

Apesar de seus benefícios, a TCI foi citada por alguns estudos como de difícil implementação, visto que muitos indivíduos relataram incômodos relacionados ao uso excessivo da contensão e ao tempo prolongado das sessões. Dessa forma, diversos autores modificaram o protocolo da terapia a fim de aumentar a aceitabilidade e adesão à mesma (DROMERICK et al., 2000; WU et al., 2007; LIN et al., 2009; PAGE et al., 2004; PAGE et al., 2008; BROGÅRDH et al., 2009; BOAKE et al., 2007; MYINT et al., 2008; MYINT et al., 2008; TAUB et al.,2005; BROGÅRDH et al., 2010). Wu et al. (2007) reduziram o tempo da contensão para 5 horas e o tempo de intervenção fisioterapêutica para duas horas diárias durante três semanas com frequência de cinco vezes por semana (WU et al., 2007; CHEN et al., 2007). Seus resultados mostraram efeitos significativos a favor do grupo $\mathrm{TCl}$. Os pacientes melhoraram em vários aspectos da função motora e da qualidade de vida (WU et al., 2007; CHEN et al., 2007). 
Alguns desses estudos, além de reduzirem o tempo de contensão do membro menos comprometido, prolongaram a aplicação da intervenção por um período de dez semanas (DROMERICK et al., 2000; PAGE et al., 2004; PAGE et al., 2008). Page et al. (2008) utilizaram a contensão nos participantes de seu estudo por cinco horas diárias durante cinco dias da semana. Por meio da realização de sessões de trinta minutos, três dias semanais, comprovaram que a TCl é eficaz no tratamento de déficits motores do membro superior de indivíduos pós-AVE, uma vez que promove aumento do uso e das habilidades motoras do membro mais comprometido (PAGE et al., 2008).

Todos os artigos selecionados nessa revisão utilizaram instrumentos padronizados de avaliação antes e após a aplicação das intervenções como um parâmetro para avaliar os resultados e a evolução do tratamento (WOLF et al., 2006; PAGE et al., 2009). Tal fato traduz-se em dados mais objetivos e, consequentemente, em desfechos mais fidedignos e sensíveis à população estudada.

\section{CONCLUSÃO}

A Terapia por Contensão Induzida mostrou-se eficaz em pacientes com Acidente Vascular Encefálico que apresentam déficits funcionais de membro superior. Sugere-se, quando utilizada a referida técnica, a contensão do membro menos comprometido durante $90 \%$ do dia e fisioterapia diária de 4 horas para o membro mais comprometido com treino intenso e repetitivo de atividades funcionais durante duas semanas consecutivas.

\section{REFERÊNCIAS}

BRANDÃO, M. B.. Efeitos da terapia de movimento induzido por restrição na funcionalidade de crianças com paralisia cerebral. Universidade Federal de Minas Gerais, 2007.

BOAKE, C.; NOSER, E. A.; RO, T.; BARANIUK, S.; GABER, M.; JOHNSON, R.. Constraint-Induced Movement Therapy During Early Stroke Rehabilitation. Neurorehabil Neural Repair, v.21, n.1, p.14-24, 2007.

BRANDÃO, M. B.; MANCINI, M. C.; VAZ, D. V.; BUENO, A. M.; FURTADO, S. R. C.; COELHO, Z. A. C.. Effects of constraintinduced movement therapy in children with hemiplegia: a single case experimental study. Rev. Bras. Fisioter., v.13, n.6, p.527-34, 2009.

BROGÅRDH, C.; VESTLING, M.; SJÖLUND, B. H.. Shortened constraint-induced movement therapy in subacute stroke no effect of using a restraint: a randomized controlled study with independent observers. J Rehabil Med, v.41, p.231-6, 2009.

BROGÅRDH, C.; LEXELL; J.. A 1-year follow-up after shortened constraint-induced movement therapy with and without mitt poststroke. Arch Phys Med Rehabil, v.91, p.460-4, 2010.

CARVALHO, A. C.; VANDERLEl; L. C. M.; BOFI, T. C.; PEREIRA, J. D. A. S.; NAWA, V. A.. Projeto Hemiplegia - Um modelo de fisioterapia em grupo para hemiplégicos crônicos. Arq Ciênc Saúde, v.14, n.3, p.161-8, 2007.
DAHL, A.E.; ASKIM, T.; STOCK, R.; LANGORGEN, E.; LYDERSEN, S.; INDREDAVIK, B.. Short and long-term outcome of constraint-induced movement therapy after stroke: a randomized controlled feasibility trial. Clin Rehabil. v.22, n.5, p.436-447, 2008.

DROMERICK, A.W.; LANG, C.E.; BIRKENMEIER, R.L.; WAGNER, J.M.; MILLER, J.P.; VIDEEN, T. O.. Very Early Constraint-Induced Movement during Stroke Rehabilitation (VECTORS): A single-center RCT. Neurology v.73, p.195-201, 2009.

DROMERICK, A.W.; EDWARDS, D.F.; HAHN, M.. Does the Application of Constraint-Induced Movement Therapy During Acute Rehabilitation Reduce Arm Impairment After Ischemic Stroke? Stroke v.31, p.2984-8, 2000.

ESCARCEL, B. W.; MÜLLER, M.R.; RABUSKE, M.. Análise do controle postural de pacientes com AVC Isquêmico próximo a alta hospitalar. Rev Neurocienc v.18, n.4, p.498-504, 2010.

FESS, E. E.. Grip Strength. Clinical assessment recommendations. 2 ed. Chicago: American Society of Hand Therapists, 1992.

FARIA, I.. Função do membro superior em hemiparéticos crônicos: Análise através da Classificação Internacional de Funcionalidade, Incapacidade e Saúde. Universidade Federal de Minas Gerais, 2008. 
GAUTHIER, L.V.; TAUB, E.; MARK, V.W.; PERKINS, C.; USWATTE, G.. Improvement After Constraint-Induced Movement Therapy Is Independent of Infarct Location in Chronic Stroke Patients. Stroke v.40, p.2468-2472, 2009.

LIN, K.C.; CHANG, Y.F.; WU, C.Y.; CHEN, Y.A.. Effects of Constraint-Induced Therapy Versus Bilateral Arm Training on Motor Performance, Daily Functions, and Quality of Life in Stroke Survivors. Neurorehabil Neural Repair. v.23, n.5, p.441-8, 2009.

LEITE, N. N.; BORBA, A.D.O.; SILVA, M.J.; NASCIMENTO, N.S.; SILVA, N.A.; CONCEIÇÃO, E.C.G.. Uso da bola terapêutica no equilíbrio estático e dinâmico de pacientes com hemiparesia. Fisioter. Mov. v.22, n.1, p.121-131, 2009.

MARCOTTI, A. C.. Estudo dos efeitos da dominância hemisférica cerebral esquerda sobre o acidente vascular encefálico através de três escalas de funcionalidade. Centro de Ciências Biológicas e da Saúde da Universidade Estadual do Oeste do Paraná - Campus Cascavel, 2005.

MAKI, T.. Toxina botulínica tipo A e estimulação elétrica funcional no membro superior de pacientes crônicos pósacidente vascular cerebral. Faculdade de Ciências Médicas da Universidade Estadual de Campinas, 2005.

MACEDO, A. S.. A eficiência do treino de marcha na esteira com suporte parcial de peso nacional em pacientes hemiparéticos crônicos pós Acidente Vascular Cerebral. Faculdade de Ciências Médicas da Universidade Estadual de Campinas, 2008.

MYINT, M.W.W.; YUEN, F.C.; YU, K.K.; KNG, P.L.; WONG, M.Y.; CHOW, K.C.. A study of constraint-induced movement therapy in subacute stroke patients in Hong Kong. Clin Rehabil. v.22, p.112-24, 2008.

MYINT, M.W.W.; YUEN, F.C.; YU, K.K.; KNG, P.L.; WONG, M.Y.; CHOW, K.C.. Use of constraint-induced movement therapy in Chinese stroke patients during the sub-acute period. Hong Kong Med J v.14, n.5, p.40-2, 2008.

MENEGHETTI, C.H.Z.; SILVA, J.A.; GUEDES, C.A.V.. Terapia de restrição e indução ao movimento no paciente com AVC: relato de caso. Rev Neurocienc v.18, n.1, p.18-23, 2010.

NADEAU, S. E.; BEHRMAN, A. L.; DAVIS, S.E.; REID, K.; WU, S.S.; STIDHAM, B.S.. Donepezil as an adjuvant to constraintinduced therapy for upper-limb dysfunction after stroke: An exploratory randomized clinical trial. JRRD v.41, n.4, p.525534, 2004.

PAGE, S.J.; SISTO, S.A.; LEVINE, P.; MCGRATH, R.E.. Efficacy of Modified Constraint-Induced Movement Therapy in Chronic Stroke: A Single-Blinded Randomized Controlled Trial. Arch Phys Med Rehabil v.85, p.14-8, 2004.

PAZ, L.P.S.. Quantidade e qualidade do uso da extremidade superior parética após acidente cerebrovascular. Faculdade de Ciências Médicas da Universidade Estadual de Campinas, 2007.

PAZ, L.P.S; BORGES, G.. Teste da Ação da Extremidade Superior como medida de comprometimento após AVC. Rev Neurocienc v.15, n.4, p.277-283, 2007.
PAGE, S.J.; LEVINE, P.; LEONARD, A.; SZAFLARSKI, J.P.; KISSELA, B.M.. Modified Constraint-Induced Therapy in Chronic Stroke: Results of a Single-Blinded Randomized Controlled Trial. Phys Ther. v.88, n.3, p.333-40, 2008.

PAGE, S.J.; LEVINE, P.; KHOURY, J.C.. Modified ConstraintInduced Therapy Combined With Mental Practice: Thinking Through Better Motor Outcomes. Stroke v.40, p.551-4, 2009.

PEDro. Physiotherapy Evidence Database. Sydney: School of Physiotherapy- University of Sydney, Inc.. 2011.

RIBERTO, M.; MONROY, H. M.; KAIHAMI, H.N.; OTSUBO, P.P.S.; BATTISTELLA, L.R.. A terapia de restrição como forma de aprimoramento da função do membro superior em pacientes com hemiplegia. Acta Fisiatr v.12, n.1, p.15-19, 2005.

RICHARDS, L.; ROTHI, L. J. G.; DAVIS, S.; WU, S. S.; NADEAU, S. E.. Limited dose response to Constraint-Induced Movement Therapy in patients with chronic stroke. Clin Rehabil. v.20, p.1066-1074, 2006.

SUPUTTITADA, A.; SUWANWELA, N. C.; TUMVITEE S. Effectiveness of Constraint-induced Movement Therapy in Chronic Stroke Patients. J Med Assoc Thai v.87, n.12, p.1482-90, 2004.

SALIBA, V. A.; JÚNIOR, I. P. C; FARIA, C. D. C. M.; TEIXEIRASALMELA, L. F.. Propriedades psicométricas da Motor Activity Log: uma revisão sistemática da literatura. Fisioter. Mov. v.21, n.3, p.59-67, 2008.

SCHUSTER, R. C.; ZADRA, K.; LUCIANO, M.; POLESE, J. C.; MAZZOLA, D.; SANDER, I.. Análise da pressão plantar em pacientes com Acidente Vascular Encefálico. Rev Neurocienc v.16, n.3, p.179-183, 2008.

SANTOS, J. C. C.; GIORGETTI, M. J. S.; TORELLO, E. M.; MENEGHETTI, C. H. Z.; ORDENES, I. E. U.. A influência da Kinesio Taping no tratamento da subluxação de ombro no Acidente Vascular Cerebral. Rev Neurocienc v.18, n.3, p.335$340,2010$.

SILVA, L. A.; TAMASHIRO, V.; ASSIS, R. D.. Terapia por contensão induzida: revisão de ensaios clínicos. Fisioter. Mov. v.23, n.1, p.153-9, 2010.

TEIXEIRA-SALMELA, L. F.; OLIVEIRA, E. S. G.; SANTANA, E. G. S.; RESENDE, G. P.. Fortalecimento muscular e condicionamento físico em hemiplégicos. Acta Fisiatr v.7, n.3, p.108-118, 2000.

TORRIANI, C.; QUEIROZ, S. S.; CYRILLO, F. N.; MONTEIRO, C. B. M.; FERNANDES, S.; PADOAN, B. B.. Correlação entre transferência de peso sentado e alteração sensorial em região glútea em pacientes hemiplégicos/paréticos. Rev Neurocienc v.13, n.3, p.117-121, 2005.

TEIXEIRA-SALMELA, L. F.; LIMA, R. C. M.; LIMA, L. A. O.; MORAIS, S. G.; GOULART, F.. Assimetria e desempenho funcional em hemiplégicos crônicos antes e após programa de treinamento em academia. Rev. Bras. Fisioter. v.9, n.2, p.227-233, 2005 
TAUB, E.; LUM, P. S.; HARDIN, P.; MARK, V. W.; USWATTE, G.. AutoCITE: Automated Delivery of $\mathrm{Cl}$ Therapy With Reduced Effort by Therapists. Stroke v.36, p.1301-4, 2005.

TAUB, E.; USWATTE, G.; KING, D. K.; MORRIS, D.; CRAGO, J. E.; CHATTERJEE, A.. A Placebo-Controlled Trial of ConstraintInduced Movement Therapy for Upper Extremity After Stroke. Stroke v.37, p.1045-9, 2006.

TORRIANI, C.; MOTA, E. P. O.; SALES, A. L. M.; RICCI, M.; NISHIDA, P.; MARQUES, L.. Efeitos da estimulação motora e sensorial no pé de pacientes hemiparéticos pós Acidente Vascular Encefálico. Rev Neurocienc v.16, n.1, p.25-9, 2008.

UNDERWOOD, J.; CLARK, P. C.; BLANTON, S.; AYCOCK, D. M.; WOLF, S. L.. Pain, Fatigue, and Intensity of Practice in People With Stroke Who Are Receiving Constraint-Induced Movement Therapy. Phys Ther. v.86, n.9, p.1241-1250, 2006.

WITTENBERG, G. F.; CHEN, R.; ISHII, K.; BUSHARA, K. O.; TAUB, E.; GERBER, L. H.. Constraint-Induced Therapy in Stroke: Magnetic-Stimulation Motor Maps and Cerebral Activation. Neurorehabil Neural Repair v.17, n.1, p.48-57, 2003.

WINSTEIN, C. J.; MILLER J. P.; BLANTON, S.; TAUB, E.; USWATTE, G.; MORRIS, D.. Methods for a Multisite Randomized Trial to Investigate the Effect of ConstraintInduced Movement Therapy in Improving Upper Extremity Function among Adults Recovering from a Cerebrovascular Stroke. Neurorehabil Neural Repair v.17, n.3, p.137-152, 2003.
WOLF, S. L.; WINSTEIN, C. J.; MILLER, J. P.; TAUB, E.; USWATTE, G.; MORRIS, D.. Effect of Constraint-Induced Movement Therapy on Upper Extremity Function 3 to 9 Months After Stroke: The EXCITE Randomized Clinical Trial. JAMA. v.296, n.17, p.2095-2104, 2006.

WU, C. Y.; LIN, K. C.; CHEN, H. C.; CHEN, I. H.; HONG, W. H.. Effects of modified constraint-induced movement therapy on movement kinematics and daily function in patients with stroke: a kinematic study of motor control mechanisms. Neurorehabil Neural Repair v.21, n.5, p.460-6, 2007.

WU, C. Y.; CHEN, C. L.; TANG, S. F.; LIN, K. C.; HUANG, Y. Y. Kinematic and clinical analyses of upper-extremity movements after constraint-induced movement therapy in patients with stroke: a randomized controlled trial. Arch Phys Med Rehabil v.88, p.964-70, 2007;

WU, C. Y.; CHEN, C. L.; TSAI, W. C.; LIN, K. C.; CHOU, S. H.. A Randomized Controlled Trial of Modified Constraint-Induced Movement Therapy for Elderly Stroke Survivors: Changes in Motor Impairment, Daily Functioning, and Quality of Life. Arch Phys Med Rehabil v.88, p.273-8, 2007;

WOLF, S. L.; THOMPSON, P. A.; WINSTEIN, C. J.; MILLER, J. P.; BLANTON, S. R.; NICHOLS-LARSEN, D. S.. The EXCITE Stroke Trial. Comparing Early and Delayed Constraint-Induced Movement Therapy. Stroke, v.41, p.2309-2315, 2010.

YEN, J. G.; WANG, R. Y.; CHEN, H. H.; HONG, C. T. Effectiveness of Modified Constraint-Induced Movement Therapy on Upper Limb Function in Stroke Subjects. Acta Neurol Taiwan, v.14, n.1, p.16-20, 2005. 